\section{Impacto social del reciclaje en el futuro de la sociedad}

Social Impact of recycling on the future the society
Centro Sur.

Social Science Journa

Julio - Diciembre Vol 4 No 2

http://centrosureditorial.com/ind ex.php/revista

eISSN: 2600-5743

revistacentrosur@gmail.com

Recepción: 18 marzo 2019

Aprobación 3 abril 2020

Pag 332-341

Atribución/Reconocimiento-

NoComercial-Compartirlgual 4.0 Licencia Pública Internacional -

CC BY-NC-SA 4.0

https://creativecommons.org/lice nses/by-nc-sa/4.0/legalcode.es

\section{Resumen}

El proyecto fomentó el impacto social de la Universidad Santo Tomás, Seccional Tunja, fortaleciendo las habilidades en oficios y rescate de tradiciones ancestrales como la cestería. El objetivo principal fue la promoción del cuidado del medio ambiente a través del reciclaje y la construcción de lazos sociales. Como pregunta orientadora se planteó el ¿Qué estrategias permiten el aprovechamiento de materiales reciclados? A través de este, se buscó disminuir el daño ambiental, se trabajaron materias primas como papel periódico, revistas, cartón y plástico y su reconversión en elementos decorativos y utilitarios que promuevan el ahorro e inclusive generen algunos ingresos económicos al hogar. Como resultados del proyecto se realizaron: muestra de exposición y venta de productos, incentivando la creación de unidades productivas, divulgación en redes sociales y radio, aprovechamiento del tiempo libre, $y$, en los adultos mayores activación de motricidad fina, memoria y un espacio en el cual mantenerse socialmente activo.

Palabras clave: Desarrollo Comunitario, Reciclaje, Rescate de Tradiciones, Responsabilidad Social Universitaria, Tejido de Lazo Social

\begin{abstract}
Magister en Pedagogía. Universidad Santo Tomás Tunja Tunja - Colombia ORCID. https://orcid.org/0000-0003-3671-2205. Email. elsa.guzman01@usantoto.edu.co.

https://scholar.google.es/citations?user=jfMSmB4A AAAJ\&hl=es

Maestría en Ciencias Matemáticas. Universidad Santo Tomás Tunja. Tunja - Colombia. Orcid. https://orcid.org/0000-0003-2742-368X E-mail: luz.pabon@usantoto.edu.co. https://scholar.google.com/citations?user=|k|EgHOA $\underline{\text { AAAJ\&hl=es }}$
\end{abstract}




\section{Abstract}

The project promoted the social impact of the Saint Tomas University, Tunja Section, strengthening skills in trades and rescue of ancestral traditions such as basketry. The main objective was the promotion of environmental care through recycling and the construction of social ties. As a guiding question, what strategies are allowed to take advantage of recycled materials? Through this, it was sought to reduce environmental damage, raw materials such as newspaper, magazines, cardboard and plastic and its conversion into decorative and utilitarian elements that promote savings and even generate some household income. As results of the project were carried out: current exhibition and sale of products, encouraging the creation of productive units, dissemination in social networks and radio, use of free time, and, in older adults activation of fine motor skills, memory and a space in which stay socially active.

Key words: Community development, Recycling, Rescue of Traditions, Social Responsibility, Develop Social Ties

\section{Introducción}

La Universidad Santo Tomás, Seccional Tunja, en el desarrollo de sus funciones sustantivas contempla la docencia, la Responsabilidad Social Universitaria (RSU) y la investigación. En la dirección de la RSU se abordan como líneas estratégicas: Egresados, Emprendimiento, Desarrollo Comunitario, Asesorías y Consultorías, Relaciones Interinstitucionales e Internacionalización. El proyecto titulado "Reciclando Ando...por un mañana mejor" responde a los Objetivos de Desarrollo Sostenible (ODS) en cuanto a la promoción y consumo responsable, ya que es una práctica en la gestión de residuos sólidos porque permite el aprovechamiento de los recursos y la reducción de los efectos ambientales (Programa de las Naciones Unidas para el Desarrollo - PNUD, 2018). Hace parte del desarrollo comunitario, acercándose a la Comunidad del Barrio Bolívar, mediante actividades que promueven el reciclaje y protección de los recursos naturales.

Vale la pena, tener en cuenta que el reciclaje es la actividad de recuperar los desechos sólidos a fin de reintegrarlos al ciclo económico, reutilizándolos o aprovechándolos como materia prima para nuevos productos, logrando beneficios económicos, sociológicos y sociales (Röben, 2003). Sin embargo, "el reciclaje no es la principal solución al problema de la basura, sino una actividad económica que se debe abordar como un elemento dentro de un conjunto de soluciones (ADAN, 1999, citado en Reyes Curcio, Pellegrini Blanco, \& Reyes Gil, 2015, pág. 160). 
Como referente legal, se cuenta con el decreto 605 de 1996, en donde, "es obligación del usuario de depositar temporalmente los residuos sólidos, mientras se procesan para su aprovechamiento" (Ministerio de Desarrollo Económico, 1996). En la Ciudad de Tunja, se cuenta con el Plan de Gestión Integral de Residuos Sólidos (PGIRS), que reconoce la labor de las asociaciones de recicladores: Recitunja, Reciboy, con la vinculación de 18 y 24 personas respectivamente, dedicados a la clasificación, selección, empaque y transporte de residuos reciclables: Papel, Cartón, Chatarra, Plástico de botella, Aluminio, Vidrio, Bronce, Cobre y Periódico, mediante rutas selectivas. De acuerdo a las cifras que el documento registra, para una muestra de $143 \mathrm{~kg}$, de ruta residencial, la cantidad de papel corresponde al 3.5\%, el cartón al $5.9 \%$ y el plástico al $19.6 \%$, material que se dispone en el Relleno Sanitario de Pirgua y, que entre residuos residenciales y comerciales corresponde a $2035.85 \mathrm{~kg}$ por día (Alcaldía Mayor de Tunja, 2015).

Es necesario, tener en cuenta que "Todas las actividades humanas, industriales, agrícolas, domésticas u otras inevitablemente producen desechos. Algunos desechos son perjudiciales para la salud. Otros, durante la descomposición, causan molestias por el olor, atraen alimañas o causan contaminación de los suelos y contaminación del agua." Situación que se está presentando en la ciudad, los sectores aledaños al Relleno Sanitario de Pirgua se están afectando por enfermedades respiratorias y la proliferación de bacterias está afectando los suelos del sector (BR, 2018).

Con el proyecto, lo que se ha logrado es el aprovechamiento de estos materiales a través de la elaboración de artículos decorativos y utilitarios para el hogar. Con el periódico y las revistas se realizan tejidos en cestería, lo cual ha permitido rescatar algunas tradiciones del departamento de Boyacá, con los cuadernos y el papel de archivo la elaboración de papel. A partir del cartón, se han elaborado muebles para el hogar, con el cartón obtenido de las cubetas de huevos y las cáscaras de los mismos, se han hecho jarrones decorativos. Y, a partir de las botellas PET se han obtenido sillas tipo puff.

En la parte social, se están beneficiando del proyecto aproximadamente 25 familias de estrato 2, quiénes entre sí, han formado una gran familia, apoyándose y compartiendo aprendizajes propios. En cuanto al colectivismo se asocia al reciclado porque relaciona el comportamiento con la satisfacción de las necesidades ajenas e incluye dimensiones semánticas antagónicas al individualismo (McCarthy y Shrum, 1994, citado en (Díaz Meneses \& Beerli Palacio, 2004). Así mismo, han sido promotores de la conciencia ambiental y de motivar cambios en sus estilos de vida, y en la disminución de desechos. Los habitantes del sector están en constante aprendizaje en búsqueda de la conservación del entorno y la utilización de los materiales existentes en nuevos y variados proyectos. 
El proyectó "Reciclando ando...por un mañana mejor" ha logrado visibilizarse en la Ciudad de Tunja mediante las redes sociales de la prensa de la Alcaldía, con la participación en ferias empresariales y a través de la difusión radial, además, de la elaboración de una cartilla paso a paso como instructivo para el desarrollo de cada uno de los elementos hasta ahora trabajados con la comunidad.

\section{Materiales y métodos}

El trabajo con la comunidad se inicia con un acercamiento a la Junta de Acción Comunal (JAC) del Barrio Bolívar, y posterior reunión de presentación de las actividades a realizar con la comunidad beneficiada. La JAC es quién convoca la comunidad del sector y presta el salón comunal para el desarrollo de los talleres. Una vez concertados los horarios se inicia con el desarrollo de los talleres que a continuación se describen:

- Charla de sensibilización: se contó con la participación de una funcionaria de la empresa SERVITUNJA quién explicó a la comunidad la necesidad de hacer separación de residuos en la fuente y la reutilización de los recursos aprovechables.

- Elaboración de papel reciclado: Con la recolección de papel de cuadernos, folios de archivo se procedió al licuado y obtención de la pulpa de papel, como materia prima para la creación de nuevo papel.

El papel reciclado disminuye la tala de árboles y el consumo de recursos, como mencionan Aguilar (2004) "Las ventajas de usar papel reciclado son obvias: se talan menos árboles y se ahorra energía. En efecto, para fabricar papel a partir de celulosa virgen se requiere madera, agua y energía; para obtener la misma cantidad con papel usado recuperado se necesita 100 veces menos cantidad de agua y una tercera parte de energía, y no se consume madera de los bosques."

Por otro, lado dentro de las bondades del papel reciclado esta la sustitución de la celulosa de los árboles por fibras provenientes de desechos agrícolas, como cáscaras, bagazo, tusa, u otras plantas (Moreno, Robayo Quintana, Ferrucho Rodríguez , \& Vargas Oyola, 2016). Así mismo, "El papel artesanal o a mano puede ser liso ó presentar diferentes texturas que se logran incluyendo en su preparación, diversas fibras naturales o procesadas, hilos, hojas secas, pétalos, flores, imágenes y otros materiales que lo enriquecen" (Aguilar Rivera, Houbron, Rustrian, \& Reyes-Alvarado, 2014, pág. 104). Alterno se elaboraron bastidores con tubos y madera de guacales, y para reemplazar la seda se utilizó 
medias veladas. Para la elaboración del papel se siguió la guía de (Salvador Eugenio, 2004).

- Láminas de papel y empaques: Con hojas de papel periódico (diarios nacionales), engrudo y fibras decorativas se hicieron láminas resistentes, que fueron utilizadas en empaques y bolsas de regalo, cumpliendo con las características de un empaque convencional, y permitiendo un desarrollo sustentable, según informe Brundtland, de 1987, se debe "satisfacer las necesidades de la generación presente sin comprometer la capacidad de las generaciones futuras de satisfacer sus propias necesidades" (Rivera, 2019, pág. 3).

- Cestería ecológica: En el Departamento de Boyacá es tradición la tejeduría a partir de fibras naturales como el esparto, fibra que es extraída de la naturaleza, tinturada de forma vagetal, secada y tratada manualmente. "Según las artesanas más veteranas este ofi- cio viene de las comunidades muiscas, aunque otras dicen que lo trajeron los españoles, cuestión que parece menos probable, pero que igual se ha enquistado en el saber popular" (Villamil Rodriguez, Gutierrez Gonzalez , \& Rodriguez Aldemar, 2020). La idea del proyecto fue reemplazar las fibras naturales a partir de las hojas de revistas y periódico, previamente cortados, con la ayuda de un alambre o un palo de pincho se realizan los rollitos de papel que se tejen según el diseño o artículo elegido. Es de resaltar, que "la cestería y los oficios textiles han estado generalmente ligados o asociados al género femenino. Las mujeres han asumido la conservación y transmisión de estos conocimientos, manteniendo viva la tradición con su saber y quehacer cotidiano" (Bustos, 1994)

- Elaboración de pulpa de cartón: Con cubetas de huevo, seleccionadas por color, una vez dejadas en agua por varios días, se muele o se tritura para obtener una pasta resistente que puede ser usada para la elaboración de macetas, decoración de frascos y latas de diferentes productos alimenticios y de aseo. Además, esta puede ser usada en la elaboración de máscaras y atuendos.

- Elaboración de muebles en cartón: A partir de cajas de cartón corrugado reciclado y engrudo se elaboran láminas resistentes que semejan aglomerados de madera, con los cuales se construyen mobiliarios que contribuyen a la economía de los hogares. Tales como zapateros, mesas de noche, esquineros, armarios, de acuerdo, a las necesidades particulares de las familias. Es de tener en cuenta que "el mobiliario que viste los espacios debe ser ideado, diseñado y estructurado con una finalidad de cumplir una función específica de acuerdo a las necesidades de los individuos" (Pineda Medina \& Roncancio Saenz, 2017). 
- Jarrones decorativos: A partir de materiales como bombas, tubos de cartón del papel de cocina, del papel higiénico, botellas PET, papel periódico y pulpa de cartón de huevos se elaboran estos elementos.

Con los productos elaborados la comunidad participó en una feria de emprendedores en donde tuvieron la oportunidad exponer y comercializar los productos elaborados. De esta manera, "emprender el negocio del reciclaje, aparte de actuar en beneficio de la humanidad y la preservación del medio ambiente, también representa un negocio rentable para la comunidad" (Sanmartín Ramón, Zhigue Luna, \& Alaña Castillo, 2017, pág. 39). Con esta actividad se lograron resultados favorables, en cuanto a la admiración por los trabajos realizados, también se notó el desconocimiento que se tiene en la ciudad del uso que se le puede dar al cartón, el papel y a las botellas. Lo cual también motiva que otras comunidades inicien actividades no solo de reciclaje sino de transformación de recursos y disminución en la contaminación.

\section{Resultados}

A partir del desarrollo del proyecto de RSU "Reciclando Ando... por un mañana mejor" se han obtenidos resultados generales, como:

1. Las familias beneficiadas expresan que han disminuido la generación de desechos y de basuras, $y$ han incentivado esta conciencia ambiental a sus conocidos y familiares, acuden a los negocios locales para la recolección del cartón, a los edificios para conseguir periódicos, a las tiendas para encargar botellas, cubetas de huevos y demás materiales que se les pueda dar una segunda oportunidad.

2. Las docentes que lideran el proyecto, promueven en la Universidad Santo Tomás Tunja, la recolección de materiales como botellas de gaseosa, revistas, papel de archivo. Con el desarrollo del proyecto algunos funcionarios de la Universidad almacenan y reciclan los materiales para que se hagan llegar a la comunidad y puedan ser transformados.

3. A través del proyecto, se han vinculado paulatinamente estudiantes de la Seccional, quienes han indagado sobre los procesos que podrían mejorar el desarrollo de los productos.

En cuanto a la elaboración de productos, se han diversificado los usos de las materias primas y se ha logrado una mayor resistencia y durabilidad de los productos, investigando sobre algunos productos químicos que brindan un mejor acabado, impermeabilidad y resistencia. Vale la pena recordar que "al diseñar objetos, cuya materia prima son los desechos, se incentiva a reciclar y optimizar el uso de los recursos; al tiempo que al ser manufacturados de forma artesanal se contribuye al desarrollo cultural y económico de la sociedad" (Alvarado Cordero, 2006, pág. 6). Al respecto, se destacan habilidades como: 
- Elaboración de diferentes muestras en los tejidos.

- Optimización del tiempo en la manufactura de los productos.

- En la elaboración de los productos se han reducido costos con la elaboración de pegamentos y barnices caseros.

- Los productos obtenidos son únicos y son producto de la creatividad y experiencia personal.

- En los productos, gracias a la creatividad y el ingenio de quien los elabora, se ha explorado la combinación de diferentes materiales.

Gracias a las muestras y exposiciones los beneficiados del proyecto han desarrollado técnicas de atención al cliente, lo que les ha permitido dar a conocer sus productos, la técnica de la elaboración, y el empaque y venta del mismo. Con la elaboración de los muebles en cartón, han disminuido los gastos al interior de las familias. A partir del proyecto, algunos de los asistentes, se han beneficiado económicamente con la venta de sus productos y son reconocidos por la elaboración de los mismos. Según Cerdá, (Cerda \& Khalilova, 2016, pág. 14) en el aspecto social: "reducir el desempleo urbano y de las minorías, mejorar las condiciones de trabajo, seguridad y bienestar... incrementa las oportunidades sociales y la interacción comunitaria".

Aunado a lo anterior, en cuanto a la construcción social, la comunicación entre los habitantes del sector ha mejorado considerablemente, ya que muchos, a pesar de vivir en el mismo barrio no se conocían. En este momento es una comunidad ejemplar en cuanto a:

- Interés por el aprendizaje, es notorio como cada quien desde su experiencia comparte los conocimientos que posee y se esmeran porque los demás aprendan.

- En cuanto a solidaridad, además de aprendizajes, comparten materiales y logros que cada uno ha explorado con estos.

- La comunidad tiene un alto sentido de responsabilidad, compromiso e interés por el desarrollo del proyecto, ya que, inclusive se reúnen en espacios diferentes a los acordados, con el ánimo de avanzar y terminar los productos.

- El proyecto ha mejorado la comunicación oral de los participantes, ya que se han tenido que enfrentar a públicos, a cámaras y micrófonos para entrevistas, lo que antes les causaba miedo.

- La vinculación al proyecto, no ha sido sólo de los que asisten a los talleres, sino del núcleo familiar. Es común que esposos y familiares se vean involucrados con el proceso, bien desde el reciclaje o desde la preparación de los materiales.

Cabe resaltar, la vinculación que se ha tenido de adultos mayores al proyecto, quiénes han encontrado un ambiente solidario de aprendizaje y para compartir su experiencia de vida. Para ellos, no sólo se trata de una tarde de distracción o de taller, sino una oportunidad para mantenerse activos. En sus rostros se refleja la satisfacción de poder apoyar a sus nietos en la elaboración de tareas, en poder elaborar detalles o regalos 
significativos para sus hijos. La elaboración de los productos ha reactivado la motricidad fina, la concentración y la memoria. Teniendo en cuenta que la elaboración de la mayoría de los productos es repetitiva.

Al respecto, estudios manifiestan que hay una correlación positiva, en cuanto a que las personas de mayor edad son las que están más comprometidas con el reciclado (Vining y Ebreo, 1990; Folz y Hazlett, 1991; Lansana, 1992 et al, citado en Díaz Meneses, Beerli Palacio, \& Martín Santana). A su vez, (Scott, 1999, citado en Aguilar Luzón, 2006) las personas de mayor edad reciclan más que los jóvenes, mientras que los jóvenes se implican mas en conductas de reciclaje a cambio de dinero.

A nivel institucional, como resultados de divulgación del proyecto se elaboró una cartilla paso a paso de algunos de los productos que se pueden obtener a partir del reciclaje e ideas de otros que se pueden realizar. Se ha hecho divulgación a través de radio y videos en redes sociales.

\section{Discusión}

Con el desarrollo del proyecto el Departamento de Ciencias Básicas realza el compromiso social de la Universidad Santo Tomás Tunja con el medio ambiente y con el desarrollo comunitario en la ciudad. ¿Qué otras alternativas desde las instituciones educativas se podrían liderar?, ¿Cómo institucionalizar a nivel municipal o departamental este tipo de estrategias en pro del medio ambiente?

A través de esta iniciativa que permitió llegar a este sector de la ciudad, buscando el mejoramiento de la calidad de vida de la población y la reducción del impacto al medio ambiente, ¿Qué otros materiales o recursos se pueden optimizar de forma artesanal?

A través de la divulgación del proyecto se vincularon voluntariamente empresas como RECITUNJA quienes apoyaron con la realización de talleres, a su vez, se contó con la asesoría de un diseñador industrial y un artesano de la ciudad de Tunja, quienes compartieron sus conocimientos e hicieron valiosos aportes a la comunidad y al proyecto, queda pendiente las pruebas de calidad que se le pueden aplicar a los productos, resistencia, impermeabilidad, quizás segunda fase del proyecto.

En la manufactura de los productos se resalta la creatividad e ingenio en los terminados y decorados de los mismos. Y, Con el proyecto se generó un espacio de concientización y sensibilización para que la comunidad se reuniera entorno al reciclaje y la protección del medio ambiente, ¿Quá tanto rehunda el trabajo comunitario en la construcción de paz de nuestro país? 
En la comunidad del Barrio Bolívar no sólo se tejieron canastos, se tejieron lazos de amistad, solidaridad, comunicación y respeto entre los habitantes del sector.

\section{Conclusiones}

\section{FALTA CONCLUSIONES.}

\section{Referencia}

Aguilar Luzòn, M. (2006). Predicción de la conducta de reciclaje a partir de la teoría de la conducta planificada y desde el modelo del valor, normas y creencias hacia el medio ambiente. Tesis Doctoral, Granada.

Aguilar Rivera, N. (2004). El reciclado de papel y cartón. Elementos 53, 54-56.

Aguilar Rivera, N., Houbron, E., Rustrian, E., \& Reyes-Alvarado, L. (Junio de 2014). PAPEL AMATE DE PULPA DE CAFÉ (Coffea arabica) (RESIDUO DE BENEFICIO HÚMEDO). Ra Ximhai, 10(3), 103-117. Recuperado el 3 de 03 de 2020, de http://www.redalyc.org/articulo.oa?id=46131111008

Alcaldía Mayor de Tunja. (2015). Organización para la actualización del PGIRS. Tunja.

Alvarado Cordero, M. I. (2006). Diseño de objetos a partir de desechos reciclables. Revista Artesanías de América(61), 123-148. Recuperado el 03 de 03 de 2020, de http://documentacion.cidap.gob.ec:8080/handle/cidap/416

BR, M. (2018). Gestión de residuos. Springer. doi:https://doi-org.craiustadigital.usantotomas.edu.co/10.1007/978-3-319-12127-7

Bustos, M. L. (1994). Cestería y mundo femenino. Historia crítica(9), 30-35.

Cerda, E., \& Khalilova, A. (2016). Economía Circular. Estrategia y competitividad empresarial, 401, 11-20.

Díaz Meneses, G., \& Beerli Palacio, A. (Junio de 2004). La jerarquía de efectos clásica d alta involucaración para la comprensión de la conducta de reciclaje considerando los valores de los consumidores. Revista Internacional de Marketing Público y no Lucrativo, 1(1), 89-109. Recuperado el 01 de 03 de 2020

Díaz Meneses, G., Beerli Palacio, A., \& Martín Santana, J. (s.f.). El modelo de hábito de reciclado según el perfil sociodemográfico de los consumidores. Las Palmas, Gran Canaria.

Ministerio de Desarrollo Económico. (1996). Decreto 605 de 1996. Decreto, Bogotá.

Moreno, F., Robayo Quintana, M., Ferrucho Rodríguez , L., \& Vargas Oyola, M. (Marzo de 2016). APROVECHAMIENTO DE RESIDUOS VEGETALES DE PÉTALOS DE 
ROSAS, TALLOS DE GIRASOL Y VÁSTAGO DE PLÁTANO PARA LA FABRICACIÓN ARTESANAL DE PAPEL. Inventum, 71-82. Recuperado el 02 de 03 de 2020, de https://revistas.uniminuto.edu/index.php/Inventum/article/view/1444/1379

Pineda Medina, I., \& Roncancio Saenz, G. (2017). Estudio de prefactibilidad para la fabricación artesanal de mobiliario a través del reciclaje o reusó de llantas en la ciudad de Bogotá - Localidad Ciudad Bolívar. Bogotá: Corporación Universitaria Minuto de Dios.

Programa de las Naciones Unidas para el Desarrollo - PNUD. (2018). ODS en Colombia: Ios retos para el 2030. Colombia.

Reyes Curcio, A., Pellegrini Blanco, N., \& Reyes Gil, R. (Septiembre-Diciembre de 2015). El reciclaje como alternativa de manejo de los residuos sólidos en el sector minas de Baruta, Estado Miranda,Venezuela. Revista de Investigación, 39(86), 157-170. doi:https://www.redalyc.org/pdf/3761/376144131008.pdf

Rivera, C. (Mayo de 2019). Los empaques biodegradables, una respuesta a la consciencia ambiental de los consumidores. Realidad Empresarial, 7, 2-8.

Röben, E. (2003). El reciclaje, Oportunidades para reducir la generación de los desechos sólidos y reintegrar materiales recuperables en el círculo económico. Municipio de Loja. Obtenido de www.ded.org.ec

Salvador Eugenio, W. (2004). Proceso para reciclar papel paso a paso (ilustrado). Vinculando. Recuperado el 21 de 10 de 2019, de http://vinculando.org/ecologia/papel_reciclado/paso_a_paso.html

Sanmartín Ramón, G., Zhigue Luna, R., \& Alaña Castillo, T. (Enero de 2017). El reciclaje: un nicho de innovación y emprendimiento con enfoque ambientalista. Universidad y Sociedad, 36-40. Recuperado el 02 de 03 de 2020, de http://rus.ucf.edu.cu/

Villamil Rodriguez, C., Gutierrez Gonzalez , S., \& Rodriguez Aldemar, L. (2020). Memorias de oficio : Cestería Cerinza Boyacá. Bogotá, Colombia: Artesanías de Colombia. 